\title{
Shifting trends and age distribution of ESKAPEEc resistance in bloodstream infection, Southwest China, 2012-2017
}

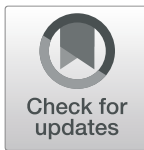

Shuangshuang Yang ${ }^{1 *}$ (D), Haofeng $\mathrm{Xu}^{2}$, Jide Sun ${ }^{1}$ and Shan Sun ${ }^{1}$

\begin{abstract}
Background: ESKAPEEC contribute to a majority of bloodstream infections (BSIs) and their antibiogram have changed overtime, while data concerning about these alterations are lacking in China. Added that a paucity of studies referred to ESKAPEEC in pediatric BSIs, our study aimed to demonstrate the longitudinal alterations of ESKAPEEc distribution and antibiogram in adult and pediatric BSIs in Southwest China.

Methods: A multicenter retrospective surveillance study was launched from 2012 to 2017. Data of China Antimicrobial Resistance Surveillance System (CARSS) was analyzed by Whonet 5.6 and Graphpad Prism 6 Software. Chi-square test or Fisher's exact test was used to examine and compare temporal changes.

Results: A total of 32,259 strains was isolated, with 17.4\% from pediatric BSIs. ESKAPEEc contributed to 58.67\% (18,924/ 32,259 ) of BSIs, with $65.3 \%$ of adult BSIs and $27.2 \%$ of pediatric BSIs. Escherichia coli (E. coli) and Klebsiella pneumoniae (K. pneumoniae) were the two predominant species. Carbapenem resistance was prevalent in 0.76, 4.60, 9.47,13.66, 59.47\% of E. coli, K. pneumoniae, Enterobacter cloacae, Pseudomonas aeruginosa (P. aeruginosa) and Acinetobacter baumannii (A. baumannii), respectively. The proportions of methicillin-resistant Staphylococcus aureus (MRSA) and vancomycinresistant Enterococcus faecium (VREFM) were 28.91\% and 2.20\%, respectively. Between 2012-2014 and 2015-2017, E. coli and $K$. pneumonia showed significantly increased resistance rates to imipenem but decreased to ceftriaxone and ceftazidime, while A. baumannii exhibited reduced resistances to almost all the beta-lactams tested. The prevalence of antimicrobial resistance to most of agents against Gram-positive ESKAPEEC did not significantly varied during the same timeframe. In comparison with those from adult BSIs, K. pneumoniae from pediatric BSIs exhibited high resistance rates to all the beta-lactams tested, especially to carbapenems (12.79\% vs 3.87\%), while A. baumannii showed low resistance rates to all the agents.
\end{abstract}

Conclusions: Ongoing burden of ESKAPEEc in BSIs and increasing trend of imipenem resistance in E. coli and K. pneumoniae call for continued surveillance. Carbapenems are still active against Gram-negative ESKAPEEc, except for A. baumannii and vancomycin or linezolid is still effective against Gram-positive ESKAPEEc. Carbapenem-resistant $K$. pneumoniae in children and carbapenem-resistant $A$. baumannii in adults necessitate effective antimicrobial strategies in consideration of age stratification.

Keywords: Bloodstream infections, ESKAPEEc, Antibiogram, Time trend, Pediatric, Adult

\footnotetext{
* Correspondence: 903878420@qq.com

'Department of Laboratory Medicine, the First Affiliated Hospital of

Chongqing Medical University, No.1, Youyi Road, Yuzhong District,

Chongqing 400016, People's Republic of China

Full list of author information is available at the end of the article
}

C The Author(s). 2019 Open Access This article is distributed under the terms of the Creative Commons Attribution 4.0 International License (http://creativecommons.org/licenses/by/4.0/), which permits unrestricted use, distribution, and reproduction in any medium, provided you give appropriate credit to the original author(s) and the source, provide a link to the Creative Commons license, and indicate if changes were made. The Creative Commons Public Domain Dedication waiver (http://creativecommons.org/publicdomain/zero/1.0/) applies to the data made available in this article, unless otherwise stated. 


\section{Background}

Sepsis has been recognized as a global health priority by world health organization (WHO) in 2017. Better assessment of antimicrobial resistance (AMR) of pathogens causing bloodstream infections (BSIs) is one of the most significant resolutions to stem the tide of BSIs and thus alleviate the burden of sepsis [1-3]. ESKAPEEc, a cluster of notorious pathogens including Enterococcus faecium, Staphylococcus aureus, Klebsiella pneumoniae, Acinetobacter baumannii, Pseudomonas aeruginosa, Enterobacter spp and Escherichia coli, have been reported to contribute $50-70 \%$ of all causative pathogens to BSIs [4-6]. The striking emergence and transmission of extensive beta-lactamases (ESBLs) or carbapenemase genes among Gram-negative robs have vigorously driven the prominence of Gram-negative ESKAPEEc in BSIs. In addition, due to the implement of vaccination campaigns and comprehensive antimicrobial stewardship activities, a shift in the distribution and AMR patterns of ESKAPEEc was ongoing worldwide [7-11]. However, data concerning about these changes in BSIs were lacking in China.

Moreover, foregoing studies failed to discuss these alterations in pediatric populations. A longitudinal surveillance study in United States indicated that children witnessed an increased prevalence of carbapenem-resistant Enterobacteriaceae (CRE) in BSIs from $0.0 \%$ in 1999-2000 to $4.5 \%$ in 2011-2012 [12]. Recently, national surveillance data from China in 2013 had reported that pediatric patients witnessed highest prevalence of carbapenem resistance in Klebsiella pneumoniae (10.6\%) [4]. However, a paucity of data has referred to the shifting of this high prevalence among pediatric BSIs over time in China.

To fill this gap, we launched a 6-year retrospective multicenter study from 2012 to 2017 to elucidate the longitudinal alterations of pathogen distributions and antibiogram of ESKAPEEc among pediatric and adult BSIs in Chongqing, Southwest China. Age distributions and AMR patterns were further compared between 2012-2014 and 2015-2017 two periods.

\section{Methods}

\section{Study design and data enrollment criteria}

This retrospective study was based on blood-culture proven BSIs and conducted in the first affiliated hospital of Chongqing Medical University, a branch of the China Antimicrobial Resistance Surveillance System (CARSS) in Southwest China, which included 54 microbiological laboratories from secondary or tertiary hospitals in Chongqing. Data of all the isolates were collected from the database of CARSS. Isolates were enrolled if specifically reported all the information including patients' age, unique patient identification number and antibiotic susceptibilities with minimal inhibitory concentration (MIC) values. According to the Clinical Laboratory Standards Institute (CLSI) M39-A4, only the first isolate was evaluated in this present study, in consideration that inclusion of multiple isolates from an individual patient who has successive blood culture series, several episodes of different BSIs during hospitalization or poly-microbial BSIs may overestimate the risk of acquiring a resistant strain $[13,14]$.

\section{Bacteria identification and antimicrobial susceptibility}

All participated laboratories followed standard procedures to fulfill blood culture, bacteria identification, and antimicrobial susceptibility testing on semi- or automated systems, according to the guidelines of CLSI. Antimicrobial susceptibilities of bacteria were interpreted by the recommendation of CLSI-M100 S27.

\section{Definitions}

Children was defined if patients were no elder than 14 years old, while adult was defined if patients were elder than 14 years old [15].

ESKAPEEc was a cluster of pathogens including Enterococcus faecium (E. faecium), Staphylococcus aureus (S. aureus), Klebsiella pneumoniae (K. pneumoniae), Acinetobacter baumannii (A. baumannii), Pseudomonas aeruginosa (P. aeruginosa), Enterobacter cloacae (E. cloacae) and Escherichia coli (E. coli) [5].

Carbapenem resistance of $K$. pneumoniae, E. cloacae or $E$. coli strains was defined as resistance to at least one of carbapenems: ertapenem (MIC $\geq 2)$, imipenem $(\mathrm{MIC} \geq 4)$ or meropenem $(\mathrm{MIC} \geq 4)$, while that of $A$. baumannii or $P$. aeruginosa strains was defined if resistance to either imipenem $(\mathrm{MIC} \geq 8)$ or meropenem $(\mathrm{MIC} \geq 8)$.

\section{Statistical analysis}

Raw data was firstly processed by Whonet 5.6 software and then calculated on Graphpad prism 6 and SPSS v.21.0 (SPSS, Chicago, IL, USA) software. Temporal changes in age distributions and AMR were further determined by Chi-square test or Fisher's exact test. Statistical significance was confirmed if a two-tailed $P$ value was no more than 0.05 .

\section{Results}

\section{Alterations of ESKAPEEc distribution in BSIs}

Between 2012 and 2017, a total of 36,809 strains was isolated from blood culture specimens. Of them, 32,259 strains were included according to the enrollment criteria (Fig. 1). ESKAPEEc accounted for 58.67\% (18,924/ $32,259)$ of Blood-borne pathogens. As shown in Table 1, E. coli $(32.03 \%)$ was the most frequently isolated bacteria, followed by K. pneumoniae (11.10\%), Staphylococcus epidermidis (10.23\%) and S. aureus (6.05\%). The 


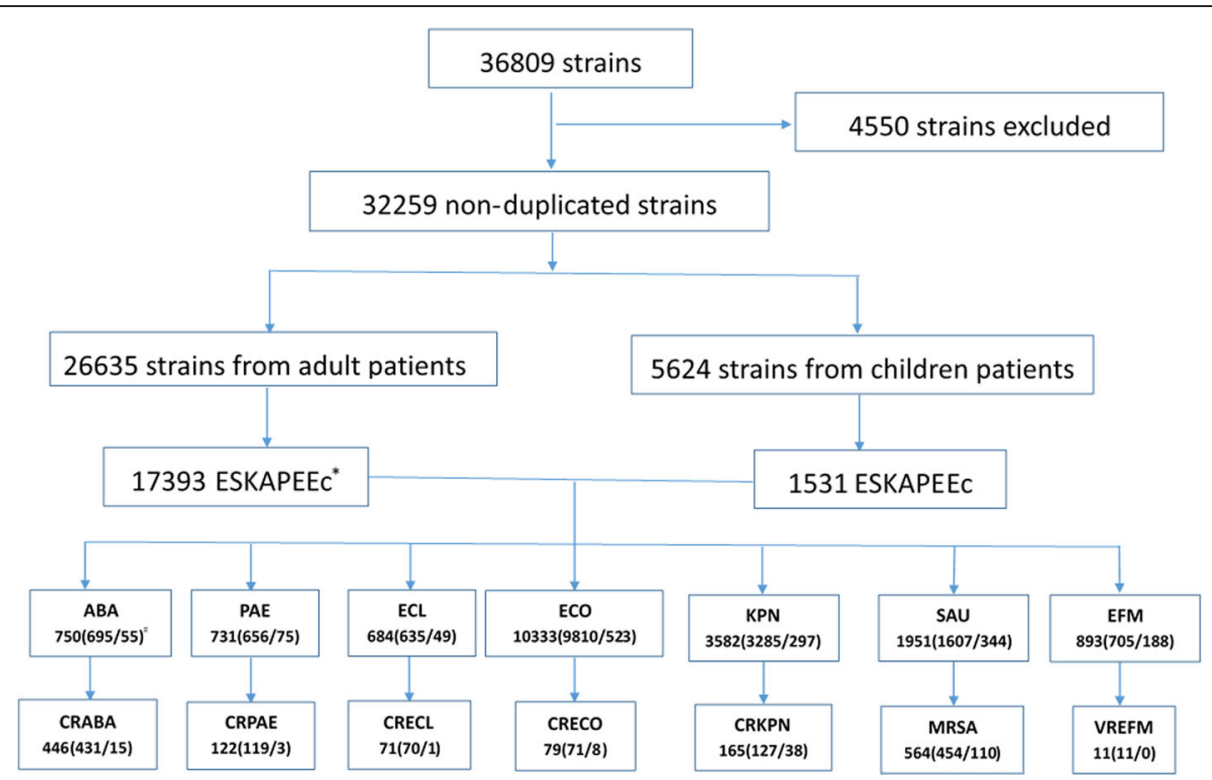

Fig. 1 ESKAPEEC pathogens encountered in blood culture specimens in this present study. ESKAPEEc*: Enterococcus faecium (EFM), Staphylococcus aureus (SAU), Klebsiella pneumoniae (KPN), Acinetobacter baumannii (ABA), Pseudomonas aeruginosa (PAE), Enterobacter cloacae (ECL) and Escherichia coli (ECO); CRABA: carbapenem-resistant Acinetobacter baumannii; CRPAE: carbapenem-resistant Pseudomonas aeruginosa; CRECL: carbapenem-resistant Enterobacter cloacae; CRECO: carbapenem-resistant Escherichia coli; CRKPN: carbapenem-resistant Klebsiella pneumoniae; MRSA: methicillin-resistant Staphylococcus aureus; VREFM: Vancomycin-resistant Enterococcus faecium. N (A/B) \#: N is the total number of strains from BSIs. A is the number of strains isolated from adult BSIs, while B is that from pediatric BSIs

total number of strains increased from 12,114 in $2012-$ 2014 to 20,145 in 2015-2017 (Fig. 2a) and the corresponding proportion of ESKAPEEc significantly increased from $57.3 \%$ in $2012-2014$ to $59.5 \%$ in $2015-2017\left(\chi^{2}=15.74\right.$, $P<.0001)$, largely due to the dramatical rise of $E$. coli (30.15\% vs $33.16 \%)$ and K. pneumoniae (10.52\% vs $11.46 \%)$ in isolation rate. During the same time frame, the percentages of $A$. baumannii (2.88\% vs $1.99 \%)$ and P. aeruginosa (3.07\% vs $2.59 \%$ ) were decreased (Fig. 2b).

ESKAPEEc were attributable to $65.3 \%$ of all causative pathogens in adult BSIs and $27.2 \%$ in pediatric BSIs, and showed different shifting patterns in adult and pediatric BSIs from 2012 to 2014 to 2015-2017. Briefly, there was a significant increase in the prevalence of ESKAPEEc in

Table 1 Age distribution of TOP 10 pathogens isolated from blood culture specimens, 2012-2017

\begin{tabular}{|c|c|c|c|c|c|c|c|c|c|}
\hline \multirow[t]{2}{*}{ Pathogens } & \multicolumn{3}{|l|}{ Overall } & \multicolumn{3}{|l|}{ Adults } & \multicolumn{3}{|l|}{ Children } \\
\hline & $\begin{array}{l}\text { NO of pathogens } \\
(n=32259)\end{array}$ & Percentage & $\overline{\text { Rank }}$ & $\begin{array}{l}\text { NO of pathogens } \\
(n=26635)\end{array}$ & Percentage & $\overline{\text { Rank }}$ & $\begin{array}{l}\text { NO of pathogens } \\
(n=5624)\end{array}$ & Percentage & Rank \\
\hline \multicolumn{10}{|l|}{ Gram-negative } \\
\hline Escherichia coli & 10333 & $32.03 \%$ & 1 & 9810 & $36.83 \%$ & 1 & 523 & $9.30 \%$ & 1 \\
\hline Klebsiella pneumoniae & 3582 & $11.10 \%$ & 2 & 3285 & $12.33 \%$ & 2 & 297 & $5.28 \%$ & 2 \\
\hline Pseudomonas aeruginosa & 893 & $2.77 \%$ & 7 & 818 & $3.07 \%$ & 3 & 75 & $1.33 \%$ & 3 \\
\hline Enterobacter cloacae & 749 & $2.32 \%$ & 9 & 700 & $2.63 \%$ & 4 & 49 & $0.87 \%$ & 5 \\
\hline Acinetobacter baumannii & 750 & $2.32 \%$ & 8 & 695 & $2.61 \%$ & 5 & 55 & $0.98 \%$ & 4 \\
\hline \multicolumn{10}{|l|}{ Gram-positive } \\
\hline Staphylococcus epidermidis & 3300 & $10.23 \%$ & 3 & 1713 & $6.43 \%$ & 1 & 1587 & $28.22 \%$ & 1 \\
\hline Staphylococcus aureus & 1951 & $6.05 \%$ & 4 & 1607 & $6.03 \%$ & 2 & 344 & $6.12 \%$ & 4 \\
\hline Staphylococcus hominis & 1924 & $5.96 \%$ & 5 & 1316 & $4.94 \%$ & 3 & 608 & $10.81 \%$ & 2 \\
\hline Staphylococcus haemolyticus & 1231 & $3.82 \%$ & 6 & 803 & $3.01 \%$ & 4 & 428 & $7.61 \%$ & 3 \\
\hline Enterococcus faecium & 687 & $2.13 \%$ & 10 & 499 & $1.87 \%$ & 5 & 188 & $3.34 \%$ & 6 \\
\hline Streptococcus pneumoniae & 486 & $1.51 \%$ & 12 & 262 & $0.98 \%$ & 13 & 224 & $3.98 \%$ & 5 \\
\hline
\end{tabular}



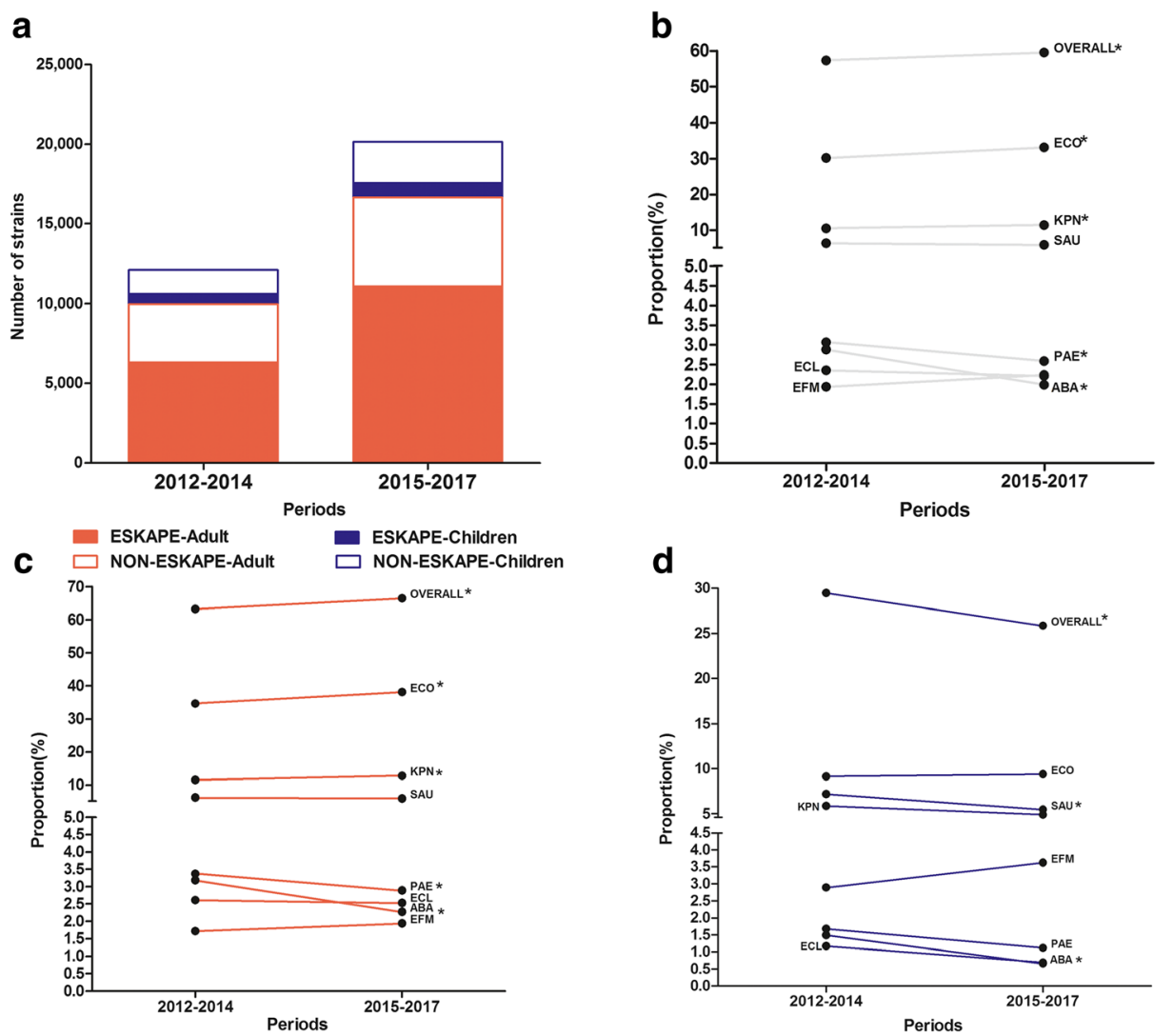

Fig. 2 Time trend and age distribution of ESKAPEEc pathogens in bloodstream infections (BSIs) from 2012 to 2014 to 2015-2017. a General distribution of ESKAPEEc pathogens in BSIs; $\mathbf{b}$ Constitutions of ESKAPEEc pathogens in BSIs; $\mathbf{c}$ Constitutions of ESKAPEEc pathogens in adult BSIs; d Constitutions of ESKAPEEc pathogens of pediatric BSIs. Asterisks indicate statistical significance as $P$ values were less than 0.05

adult BSIs $(63.23 \%$ vs $66.54 \%, P<.0001$, Fig. 2 c), which may be primarily due to the dramatically increased proportions of E. coli and K. pneumoniae. In contrast, there was a decreasing trend in pediatric BSIs $(29.46 \%$ vs $25.85 \%, P=.0035$, Fig. $2 \mathrm{~d}$ ), which may be mainly ascribed to the sharp drop in the proportions of A. baumannii and $S$. aureus during the same periods.

\section{Antimicrobial resistance (AMR) patterns of ESKAPEEc by time}

Since an increasing incidence of ESKAPEEc was observed over time, the changes in AMR profiles were further investigated. Among E. coli, the resistant rates to ceftriaxone, ceftazidime, cefepime, aztreonam, gentamicin, ciprofloxacin and levofloxacin were markedly decreased, while those to piperacillin and imipenem were dramatically increased during two studied periods from 2012-2014 to 2015-2017 (Fig. 3a). Likewise, the resistant rates of $K$. pneumoniae to ceftriaxone and ceftazidime were markedly decreased, while the resistant rates to imipenem and amikacin were significantly increased during the same periods (Fig. 3b). E. cloacae showed no significant alterations in the resistance rates to all the studied antibiotics and similar tendency was also found in $P$. aeruginosa, except for reduced resistance to ciprofloxacin (Fig. 3c \& d). Of note, A. baumannii showed a sharp reduction in the resistance to a majority of antibiotics tested, especially to imipenem (From 65.5 to $56.0 \%$ ), whereas its resistance to meropenem, amikacin and piperacillin remained stable (Fig. 3e).

As for Gram-positive ESKAPEEc, S. aureus exhibited reduced resistance to gentamicin, ciprofloxacin, erythromycin and tetracycline, while its resistance to oxacillin remained stable, near to 30\% (Fig. 3f). For E. faecium, resistance rates to all the agents tested did not significantly vary overtime, except for increased resistance to penicillin G from 58.0 to $68.9 \%$ (Fig. 3g).

\section{Antimicrobial resistance (AMR) patterns of gram-negative ESKAPEEc by age}

Since distributions of ESKAPEEc differed between adult and pediatric BSIs, we hypothesized different antibiogram of ESKAPEEc in adult and pediatric BSIs. Concerning $E$. coli, carbapenem resistance was more prevalent in pediatric BSIs than in adult BSIs. The prevalence of meropenem resistance in pediatric BSIs (2.0\%) was notably 


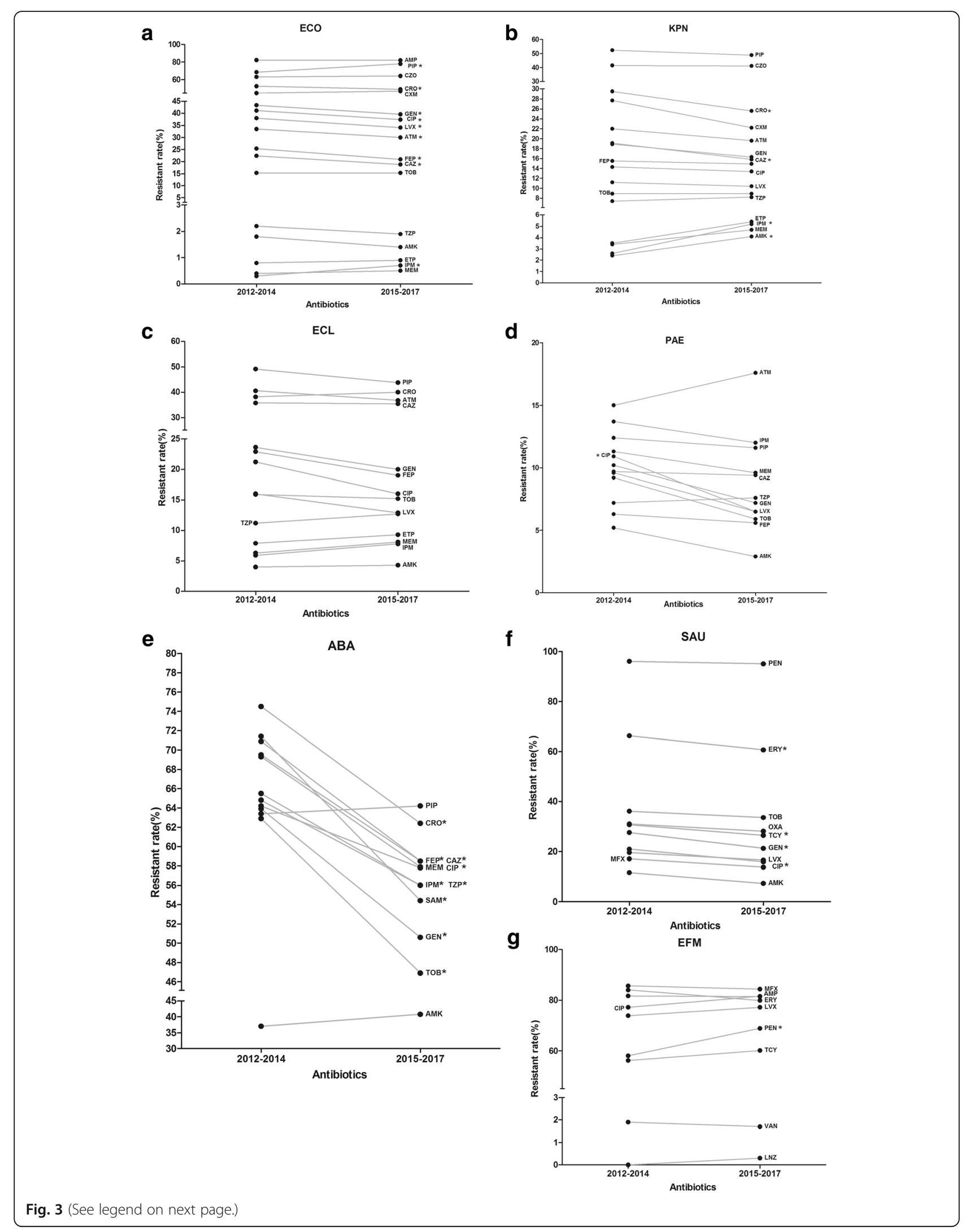


(See figure on previous page.)

Fig. 3 Alterations in antimicrobial resistance profiles of ESKAPEEc pathogens from 2012 to 2014 to 2015-2017. a ECO, Escherichia coli; b KPN, Klebsiella pneumoniae; c ECL, Enterobacter cloacae; d PAE, Pseudomonas aeruginosa; e ABA, Acinetobacter baumannii; f SAU, Staphylococcus aureus; g EFM, Enterococcus faecium. PEN: Penicillin G; AMP: Ampicillin; OXA: Oxacillin; PIP: Piperacillin; TZP: Piperacillin/Tazobactam; CZO: Cefazolin; CXM: cefuroxime; CAZ: Ceftazidime; CRO: Ceftriaxone; FEP: Cefepime; ATM: Aztreonam; ETP: Ertapenem; IMP: Imipenem; MEM: Meropenem; CIP: Ciprofloxacin; LEV: Levofloxacin; MXF: Moxifloxacin; AMK: Amikacin; GEN: Gentamicin; TOB: Tobramycin; ERY: Erythromycin; CLI: clindamycin; CHL: chloramphenicol; TCY: Tetracycline; LZD: Linezolid; VAN: Vancomycin. Asterisks indicate statistical significance as $P$ values were less than 0.05

higher than that in adult BSIs $(0.3 \%)(P<.0001$, Fig. 4a). However, resistance rates to ceftazidime and aztreonam in pediatric BSIs were lower than those in adult BSIs. No significant difference in the prevalence of ceftriaxone and cefepime resistance was observed between these two groups, ranging from 45.8 to $50.5 \%$ and 24.9 to $22.4 \%$, respectively.

In contrast to E. coli, K. pneumoniae showed different AMR profiles between these two groups. Isolates from pediatric BSIs exhibited markedly high resistance rates to all beta-lactam agents tested, compared to those from adult BSIs (Fig. 4b). In brief, 49.0 to $62.4 \%$ of isolates from pediatric BSIs, but 12.1 to $24.9 \%$ from adult BSIs were resistant to ceftriaxone, ceftazidime and cefepime. Noticeably, carbapenem resistance prevalence was $12.79 \%$ among pediatric isolates, which was higher than $3.87 \%$ among adult BSIs $(P<$. 0001).

Unlike E. coli and K. pneumoniae, no significant difference in AMR profiles of $E$. cloacae was found between adult and pediatric BSIs (Fig. 4c). Resistance rates to piperacillin/tazobartan, cefepime, amikacin, ciprofloxacin, levofloxacin and tobramycin were less than 20\%. However, it was noteworthy that carbapenem resistance among E. cloacae was $9.47 \%$.

As to non-fermenters, A. baumannii isolated from adult BSIs showed significantly higher resistance ratios to all the agents tested than those from pediatric BSIs (all $P<.05$, Fig. $4 \mathrm{~d}$ ). The prevalence of resistance to all these agents was approximately $60 \%$ in adult BSIs, especially to carbapenems (62.01\%), while that against $P$. aeruginosa was less than 20\%. Although P. aeruginosa isolated from adult BSIs showed higher resistance to almost all these antibiotics, only the prevalence of imipenem resistance was statistically higher than those from pediatric BSIs $(13.5 \%$ vs $4.2 \%, P<.05$, Fig. 4 e).

\section{Antimicrobial resistance (AMR) patterns of gram-positive ESKAPEEc by age}

In terms of Gram-positive ESKAPEEc, the prevalence of MRSA was similar among adult and pediatric BSIs (28.25\% vs $31.98 \%)$. S. aureus isolated from adult BSIs illustrated significantly higher resistance rate to moxifloxacin $(15.1 \%$ vs $5.0 \%)$, while isolates from children BSIs showed higher resistance rate to erythromycin $(59.6 \%$ vs $66.1 \%$, Fig. 4f). None of $S$. aureus isolated from either group was resistant to vancomycin or linezolid. As to $E$. faecium, resistance rates to ampicillin, ciprofloxacin, levofloxacin and moxifloxacin ranged from 69.0 to $85.9 \%$. Eleven isolates were resistant to vancomycin and all of them were isolated from adult, with a ratio of $2.20 \%$. Penicillin resistance among pediatric BSIs was $45.7 \%$, which was significantly lower than $78.5 \%$ from adult BSIs $(P<.01)$. Similar trend was also observed in levofloxacin resistance $(69.0 \%$ vs $79.2 \%, P<.05$, Fig. $4 \mathrm{~g})$.

\section{Discussions}

To our knowledge, this study presented the current available evidence of the time shifting of ESKAPEEc distributions and AMR patterns in children and adult BSIs from China. Our findings verified the increasing prevalence of ESKAPEEc in BSIs and their distinct AMR patterns in adult and pediatric BSIs.

ESKAPEEc contributed to $58.67 \%$ of BSIs, which was consistent with previous studies $[5,16]$. Our observation of an overall increasing trend of ESKAPEEc in BSIs was largely due to the striking upward trend of E. coli and $K$. pneumoniae in adult BSIs. Similar results were also concluded by a 9-year analysis of BSIs in Rome [5]. However, a recent long-term surveillance study at an urban hospital in Malawi showed a significant declination in the incidence of Enterobacteriaceae in BSIs from 1998 to 2016 [7]. This discrepancy was partly due to the heterogeneity of study design, since this present study was conducted in laboratories of more than 40 hospitals and had greater geographical representation.

Interestingly, in accordance with an 11-year retrospective study in US [17], ESKAPEEc accounted for $27.2 \%$ of all causative pathogens in pediatric BSIs, but we firstly observed a sharp drop in the prevalence of ESKAPEEc in pediatric BSIs in China, mainly driven by the slight reduction of $A$. baumannii and S. aureus. This drop may suggest that other successful species are prevalent in pediatric BSIs, but we noticed that CoNS was predominant in this present study and its predominance may override the prevalence of ESKAPEEc. Added that, data from Typhoid Surveillance in Africa Program of Sub-Saharan Africa had verified that children had significantly high odds of having a contaminated blood culture compared with adults [15]. Therefore, we deduced that blood contamination should be blamed for our high incidence of CoNS and decreased 


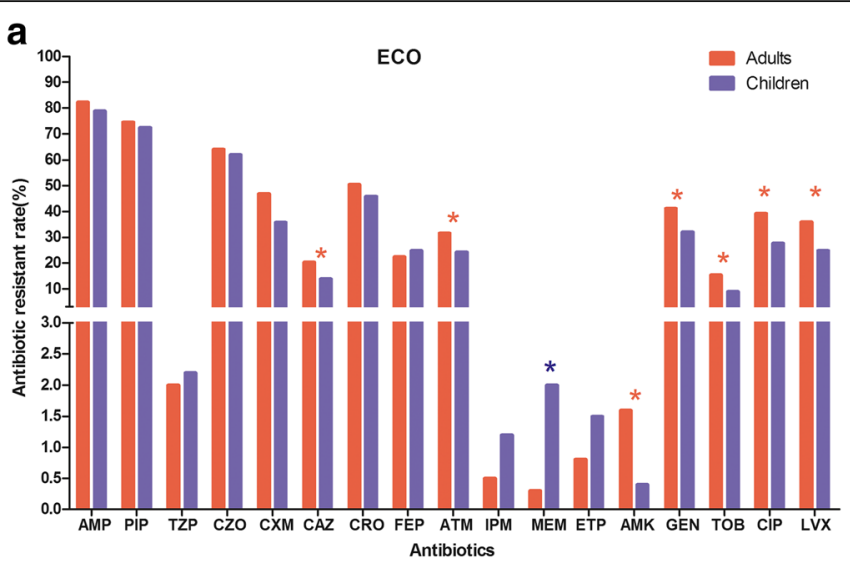

b

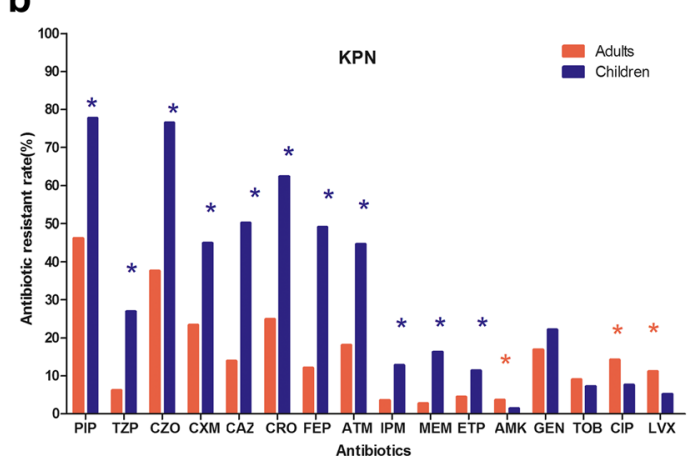

d

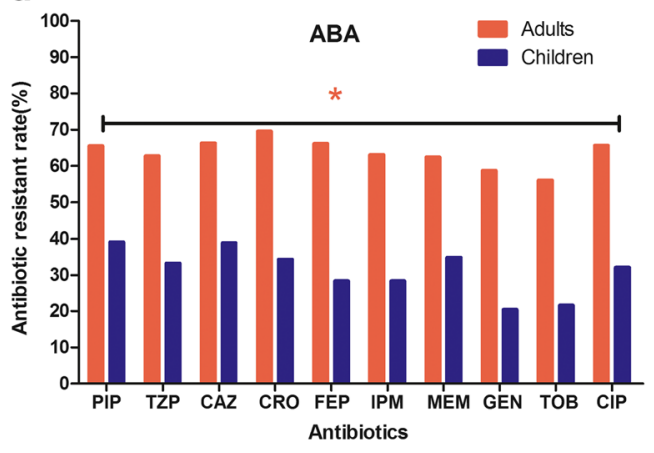

f

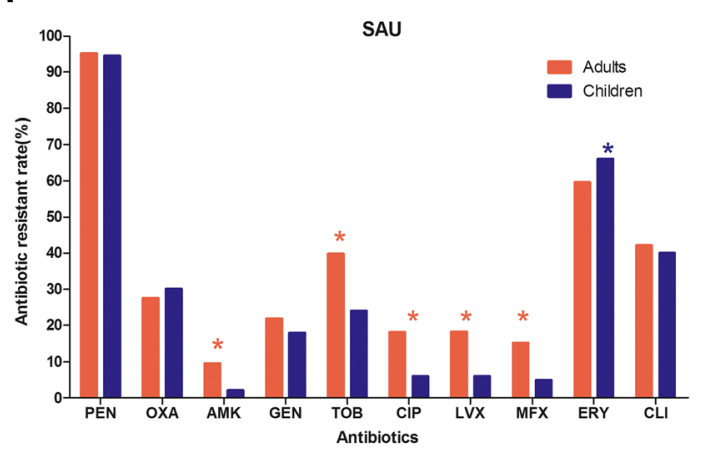

C

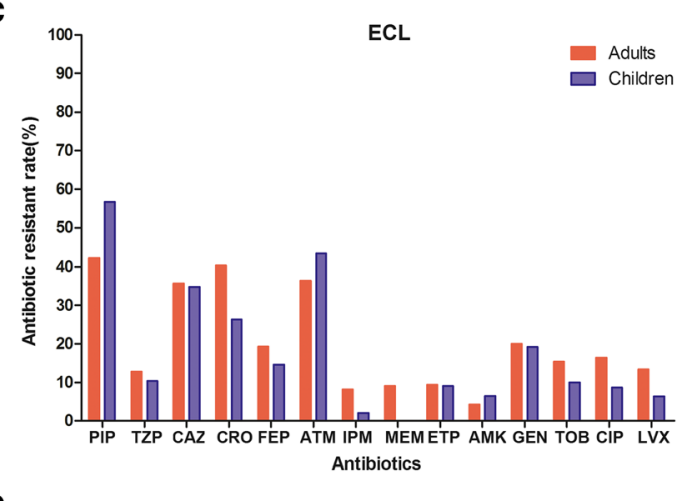

e

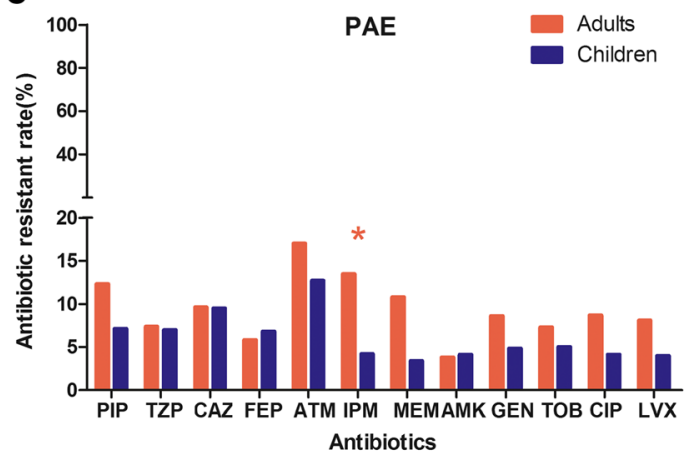

g

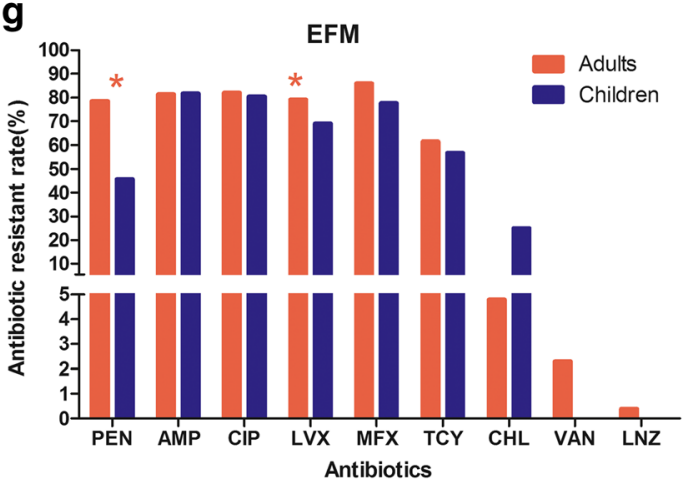

Fig. 4 (See legend on next page.) 
(See figure on previous page.)

Fig. 4 Antimicrobial resistance profiles of ESKAPEEc pathogens in adult and pediatric BSIs. a ECO, Escherichia coli; b KPN, Klebsiella pneumoniae; $\mathbf{c}$ ECL, Enterobacter cloacae; d ABA, Acinetobacter baumannii; e PAE, Pseudomonas aeruginosa; f SAU, Staphylococcus aureus; $\mathbf{g}$ EFM, Enterococcus faecium. PEN: Penicillin G; AMP: Ampicillin; OXA: Oxacillin; PIP: Piperacillin; TZP: Piperacillin/Tazobactam; CZO: Cefazolin; CXM: cefuroxime; CAZ: Ceftazidime; CRO: Ceftriaxone; FEP: Cefepime; ATM: Aztreonam; ETP: Ertapenem; IMP: Imipenem; MEM: Meropenem; CIP: Ciprofloxacin; LEV: Levofloxacin; MXF: Moxifloxacin; AMK: Amikacin; GEN: Gentamicin; TOB: Tobramycin; ERY: Erythromycin; CLI: clindamycin; CHL: chloramphenicol; TCY: Tetracycline; LZD: Linezolid; VAN: Vancomycin. Asterisks indicate statistical significance as $P$ values were less than 0.05

incidence of ESKAPEEc in pediatric BSIs, even though that previous studies in the US and Chongqing also reported CoNS was the most frequently isolated from pediatric BSIs $[17,18]$.

Unexpectedly, decreased resistance rates to ceftriaxone and ceftazidime but increased resistant rates to imipenem were observed among E. coli and K. pneumoniae. Similar trends were also observed by Lei Tian et al. [19]. The possible explanation of this alteration was that preferential antibiotic adoption of imipenem led to antibiotic selective pressure. Since ESBLs are notorious in nosocomial infection, most of empirical antibiotic therapies of BSIs are initiated with imipenem other than the third- or fourgeneration cephalosporins. Then, high consumption leads to high resistance. Recent studies in China and abroad have demonstrated that increased usage of carbapenems accelerates the production of carbapenemase, and then high proportion of CRKP [20-22].

Of note, our study alarmed high prevalence of CRKP $(12.79 \%)$ among pediatric BSIs, which was higher than data reported by CHINET [4]. However, a comparative study of neonatal BSIs in Chongqing revealed that all of 49 strains were susceptible to imipenem [18]. Our high prevalence may due to the heterogeneity of resistant element among strains and the dissemination of conservative mobile elements. Nationwide surveillance of CRE in China had confirmed carbapenemase production was the primary mechanism of carbapenem resistance in CRE. The mobile element of ISKpn27-blaKPC-2-ISKpn2 played an important role in CRKP transmission [23]. However, in pediatric patients, several studies have reported the predominance or outbreak of bla $a_{\mathrm{NDM}-1}$ among CRKP in Beijing, Shanghai and Shandong [24-26]. Our previous studies also demonstrated a high prevalence of $b l a_{\mathrm{NDM}-1}$ among CRKP in Chongqing [27, 28]. Therefore, more researches are ongoing to uncover the underlying molecular mechanisms of the high prevalence of CRKP in children BSIs and cautious adoption of carbapenems to fight against pediatric CRKP BSI is advocated.

The prevalence of carbapenem resistance among E. coli was $0.76 \%$, which was higher than results from an epicenter of CRE in the US with a ratio of 0.1\% [29]. Moreover, it was noteworthy that eight CRECO strains were isolated from pediatric BSIs, suggesting the emergence of carbapenem resistance phenotypes among pediatric inpatients in Chongqing. Despite this, carbapenems or piperacillin/ tazobactam are still active in our settings; less than 3\% strains were resistant to them.

Accompanied with declined proportion of A. baumannii in BSIs, reduced resistance rates to most of antibiotics were also observed, especially to carbapenems (From 63.61 to 55.86\%). This rate of carbapenem resistance was lower than our previous study suggesting $71.19 \%$ of CRAB in BSIs [30], but similar decreasing trend was reported in Dhaka [9]. This reduction may be owing to the implementation of CRASS strategies to fight against carbapenem-resistant robs, such as resistance surveillance, colonization clearance and environmental monitoring. However, it was noticed that in spite of this decreasing trend, resistance rates of $A$. baumannii to all these antibiotics were $60 \%$ in adult BSIs and $25 \%$ in pediatric BSIs, respectively, hence, effective treatments to fight against $A$. baumannii BSIs are still in urgent need. Recently, Tigecycline Evaluation and Surveillance Trial (TEST) concerning global blood-borne pathogens reported $70.8 \%$ of A. baumannii were susceptible to minocycline and $\mathrm{MIC}_{90}$ of tigecycline against it was $2 \mu \mathrm{g} / \mathrm{mL}$, suggesting the potential efficiencies of these two antibiotics to overcome $A$. baumannii BSIs [13].

Moreover, TEST further reported high prevalence of MRSA and VREFM worldwide, with the proportions of 33.0 and $27.6 \%$, respectively [13], while our study observed relatively low prevalence, with 28.91 and $2.2 \%$, respectively. In contradistinction to the sharp reduction of MRSA in US [10], our finding suggested the proportion of MRSA remains constant. Despite penicillin resistance was dramatically increased and ampicillin resistance was consistently high among E. faecium, vancomycin or linezolid is still efficient to fight against Gram-positive ESKAPEEc in our settings.

Several limitations should be considered in our study. Firstly, although this study included almost all the microbiology laboratories in the secondary or tertiary hospitals in Chongqing, Southwest China, it excluded some laboratories which failed to perform antimicrobial susceptibility testing. Careful interpretation of our findings was advocated in different hospital settings. Secondly, this multicenter study was focused on laboratory based, blood-culture proven BSIs attributable to 
ESKAPEEc, it, therefore, was not designed to analyze blood culture contamination and to discuss hospital-onset or community-onset without sufficient clinical patient data. Thirdly, our study excluded the isolates with antimicrobial susceptibility results reported by Kirby-Bauer, which might lead to an overestimation of the incidences of antimicrobial resistance.

\section{Conclusions}

Distributions and AMR patterns of ESKAPEEc had changed overtime and differed between adult and pediatric BSIs. We noted an ongoing burden of ESKAPEEc in BSIs, largely driven by an upward trend in adult BSIs. The two predominant species, E. coli and K. pneumoniae illustrated decreased resistances to ceftriaxone and ceftazidime but increased resistances to imipenem in the past 6 years. Carbapenems may be still efficient to fight against E. coli- and K. pneumoniae- BSIs, but it is noteworthy that high prevalence of CRKP in pediatric BSIs may wreck their efficiencies. Moreover, a decreasing tendency should not relax our vigilance of $C R A B$ in consideration of their high resistance to all these first-line antibiotics and minocycline or tigecycline may be helpful to treat A. baumannii BSIs. AMR profiles of Gram-positive ESKAPEEc remained stable and vancomycin or linezolid remains active against them. Further stratified by age, most of ESKAPEEc from pediatric BSIs showed lower resistance rates to the first-line antimicrobials, except for E. coli and K. pneumoniae, which exhibited higher resistance rates to meropenem than isolates from adults, suggesting that AMR patterns of ESKAPEEc are associated with age. Taking into account age during the continuous surveillance and infection control of ESKAPEEc in BSIs was necessitated in Chongqing, Southwest China.

\footnotetext{
Abbreviations

AMR: Antimicrobial resistance; BSIs: Bloodstream infections; CARSS: China Antimicrobial Resistance Surveillance System; CLSI: Clinical and Laboratory Standards Institute; CoNS: Coagulase-negative staphylococcus; CRABA: Carbapenem-resistant Acinetobacter baumannii; CRE: Carbapenemresistant Enterobacteriaceae; CRECL: Carbapenem-resistant Enterobacter cloacae; CRECO: Carbapenem-resistant Escherichia coli; CRKP: Carbapenemresistant Klebsiella pneumoniae; CRPAE: Carbapenem-resistant Pseudomonas aeruginosa; ESKAPEEc: Enterococcus faecium, Staphylococcus aureus, Klebsiella pneumoniae, Acinetobacter baumannii, Pseudomonas aeruginosa, Enterobacter spp (only Enterobacter cloacae included in this present study) and Escherichia coli; MIC: Minimum inhibitory concentration; MRSA: Methicillin-resistant Staphylococcus aureus; TEST: Tigecycline Evaluation and Surveillance Trial; VREFM: Vancomycin-resistant Enterococcus faecium; WHO: World health organization
}

\section{Antimicrobials}

PEN: Penicillin G; AMP: Ampicillin; OXA: Oxacillin; PIP: Piperacillin; TZP: Piperacillin/Tazobactam; CZO: Cefazolin; CXM: Cefuroxime; CAZ: Ceftazidime; CRO: Ceftriaxone; FEP: Cefepime; ATM: Aztreonam; ETP: Ertapenem; IMP: Imipenem; MEM: Meropenem; CIP: Ciprofloxacin; LEV: Levofloxacin; MXF: Moxifloxacin; AMK: Amikacin; GEN: Gentamicin; TOB: Tobramycin; ERY: Erythromycin; CLI: Clindamycin; CHL: Chloramphenicol: TCY: Tetracycline; LZD: Linezolid; VAN: Vancomycin

\section{Acknowledgements}

The authors would like to thank all enrolled laboratories for their participation in our branch, as well as Prof. Guoming Xie and PhD. Junjie Li for their suggestions of this submission.

\section{Funding}

This work was supported by Chongqing Science and Technology Commission Grant (cstc2016jcyjA0248).

Availability of data and materials

All the dataset of this article is available from the corresponding author if reasonably requested.

\section{Authors' contributions}

SSY designed this study, participated in statistic calculations and interpretation of data, and drafted this manuscript. SS and JDS collected the raw data of CRASS by Whonet 5.6. HFX participated in statistic calculations. All authors read and approved the final manuscript.

\section{Ethics approval and consent to participate}

Not applicable.

\section{Consent for publication}

Not applicable.

\section{Competing interests}

The authors declare that they have no competing interests.

\section{Publisher's Note}

Springer Nature remains neutral with regard to jurisdictional claims in published maps and institutional affiliations.

\section{Author details}

${ }^{1}$ Department of Laboratory Medicine, the First Affiliated Hospital of Chongqing Medical University, No.1, Youyi Road, Yuzhong District, Chongqing 400016, People's Republic of China. 'Laboratory Medicine, Chongqing Medical University, No.1, Yixueyuan Road, Yuzhong District, Chongqing 400016, People's Republic of China.

Received: 25 December 2018 Accepted: 20 February 2019 Published online: 29 March 2019

\section{References}

1. Reinhart K, Daniels R, Kissoon N, Machado FR, Schachter RD, Finfer S. Recognizing Sepsis as a Global Health priority - a WHO resolution. N Engl J Med. 2017;377(5):414-7.

2. Akova M. Epidemiology of antimicrobial resistance in bloodstream infections. Virulence. 2016 Apr 2;7(3):252-66.

3. Seymour CW, Gesten F, Prescott HC, Friedrich ME, Iwashyna TJ, Phillips GS, et al. Time to treatment and mortality during mandated emergency Care for Sepsis. N Engl J Med. 2017;376(23):2235-44.

4. Xu A, Zheng B, Xu Y, Huang Z, Zhong N, Zhuo C. National epidemiology of carbapenem-resistant and extensively drug-resistant gram-negative bacteria isolated from blood samples in China in 2013. Clin Microbiol Infect. 2016; 22(S1):S1-8.

5. Angelis G De, Fiori B, Menchinelli G, Inzeo TD, Liotti FM, Morandotti GA, et al. Incidence and antimicrobial resistance trends in bloodstream infections caused by ESKAPE and Escherichia coli at a large teaching hospital in Rome, a 9-year analysis ( 2007-2015 ). Eur J Clin Microbiol Infect Dis 2018; 37(9):1627-1636.

6. Buetti N, Marschall J, Atkinson A. Kronenberg a; Swiss Centre for Antibiotic Resistance (ANRESIS). National Bloodstream Infection Surveillance in Switzerland 2008-2014: different patterns and trends for university and community hospitals. Infect Control Hosp Epidemiol. 2016;37(9):1060-7.

7. Musicha P, Cornick JE, Bar-Zeev N, French N, Masesa C, Denis B. Trends in antimicrobial resistance in bloodstream infection isolates at a large urban hospital in Malawi (1998-2016): a surveillance study. Lancet Infect Dis. 2017; 17(10):1042-52

8. Hu FP, Guo Y, Zhu DM, Wang F, Jiang XF, Xu YC, et al. Resistance trends among clinical isolates in China reported from CHINET surveillance of bacterial resistance, 2005-2014. Clin Microbiol Infect. 2016;22(Suppl 1):S9-14. 
9. Ahmed D, Nahid MA, Sami AB, Halim F, Akter N, Sadique T. Bacterial etiology of bloodstream infections and antimicrobial resistance in Dhaka, Bangladesh, 2005-2014. Antimicrob Resist Infect Control. 2017;6:2.

10. Evans ME, Kralovic SM, Simbartl LA, Jain R, Roselle GA. Eight years of decreased methicillin-resistant staphylococcus aureus health care-associated infections associated with a veterans affairs prevention initiative. Am J Infect Control. 2017:45(1):13-6.

11. Xie J, Peters BM, Li B, Li L, Yu G, Xu Z, et al. Clinical features and antimicrobial resistance profiles of important Enterobacteriaceae pathogens in Guangzhou representative of southern China, 2001-2015. Microb Pathog. 2017;107:206-11.

12. Spaulding AB, Watson D, Dreyfus J, Heaton P, Grapentine S, Bendel-

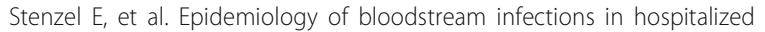
children in the United States, 2009-2016. Clin Infect Dis. 2018. https:// doi.org/10.1093/cid/ciy1030 [Epub ahead of print].

13. Zhang Z, Chen M, Yu Y, Pan S, Liu Y. Antimicrobial susceptibility among gram positive and gram-negative blood-borne pathogens collected between 2012-2016 as part of the Tigecycline evaluation and surveillance trial. Antimicrob Resist Infect Control. 2018;7:152.

14. CLSI. C.L.S.I. Analysis And Presentation Of Cumulative Antimicrobial Susceptibility Test Data: 4th Approved Guideline. CLSI document M39-A4. Wayne: CLSI; 2014

15. Nichols C, Maria L, Espinoza C, Von Kalckreuth V, Aaby P, Ahmed M, et al. Bloodstream infections and frequency of pretreatment associated with age and hospitalization status in sub-Saharan Africa. Clin Infect Dis. 2015;61(S4): S372-9.

16. El-Mahallawy HA, Hassan SS, El-Wakil M, Moneer MM. Bacteremia due to ESKAPE pathogens: an emerging problem in cancer patients. J Egypt Natl Canc Inst. 2016;28(3):157-62.

17. Larru B, Gong W, Vendetti N, Sullivan KV, Localio R, Zaoutis TE, et al. Bloodstream infections in hospitalized children: epidemiology and antimicrobial susceptibilities. Pediatr Infect Dis J. 2016;35(5):507-10.

18. Wang S, Chen S, Feng W, Sun F, Wang Q, Zhu K, et al. Clinical characteristics of nosocomial bloodstream infections in neonates in two hospitals. China J Trop Pediatr. 2018;64(3):231-6.

19. Tian L, Sun Z, Zhang Z. Antimicrobial resistance of pathogens causing nosocomial bloodstream infection in Hubei Province, China, from 2014 to 2016 : a multicenter retrospective study. BMC Public Health. 2018;18(1):1121.

20. Yang $P$, Chen $Y$, Jiang $S$, Shen $P, L U$ X, Xiao Y. Association between antibiotic consumption and the rate of carbapenem-resistant gram-negative bacteria from China based on 153 tertiary hospitals data in 2014. Antimicrob Resist Infect Control. 2018;7:137.

21. Zhang D, Hu S, Sun J, Zhang L, Dong H, Feng W. Antibiotic consumption versus the prevalence of carbapenem-resistant gram-negative bacteria at a tertiary hospital in China from 2011 to 2017. J Infect Public Health. 2018. https://doi.org/10.1016/j.jiph.2018.10.003 [Epub ahead of print].

22. Meyer E, Schwab F, Schroeren-Boersch B, Gastmeier P. Dramatic increase of third-generation cephalosporin-resistant $E$. coli in German intensive care units: secular trends in antibiotic drug use and bacterial resistance, 2001 to 2008. Crit Care. 2010;14(3):R113.

23. Zhang R, Liu L, Zhou H, Waichi E, Li J, Fang Y, et al. Nationwide surveillance of clinical Carbapenem-resistant Enterobacteriaceae (CRE) strains in China. EBioMedicine. 2017;19:98-106.

24. Jin Y, Shao C, Li J, Fan H, Bai Y, Wang Y. Outbreak of multidrug resistant NDM-1-producing Klebsiella pneumoniae from a neonatal unit in Shandong Province. China PLoS One. 2015;10(3):e0119571.

25. Zhang X, Chen D, Xu G, Huang W, Wang X. Molecular epidemiology and drug resistant mechanism in carbapenem-resistant Klebsiella pneumoniae isolated from pediatric patients in Shanghai, China. PLoS One. 2018;13(3): e0194000.

26. Huang $X$, Cheng $X$, Sun P, Tang C, Ni F, Liu G. Characteristics of NDM-1producing Klebsiella pneumoniae ST234 and ST1412 isolates spread in a neonatal unit. BMC Microbiol. 2018;18(1):186

27. Yan J, Pu S, Jia X, Xu X, Yang S, Shi J, et al. Multidrug resistance mechanisms of Carbapenem resistant Klebsiella pneumoniae strains isolated in Chongqing, China. Ann Lab Med. 2017;37(5):398-407.

28. Tian X, Sun S, Jia X, Zou H, Li S, Zhang L. Epidemiology of and risk factors for infection with extended-spectrum $\beta$-lactamase-producing carbapenemresistant Enterobacteriaceae: results of a double case-control study. Infect Drug Resist. 2018;11:1339-46.
29. Satlin MJ, Chen L, Patel G, Gomez-Simmonds A, Weston G, Kim AC, et al. Multicenter Clinical and Molecular Epidemiological Analysis of Bacteremia Due to Carbapenem-Resistant Enterobacteriaceae (CRE) in the CRE Epicenter of the United States. Antimicrob Agents Chemother. 2017;61(4).

30. Yang S, Sun J, Wu X, Zhang L. Determinants of Mortality in Patients with Nosocomial Acinetobacter baumannii Bacteremia in Southwest China: A Five-Year Case-Control Study. Can J Infect Dis Med Microbiol. 2018;2018: 3150965 .
Ready to submit your research? Choose BMC and benefit from:

- fast, convenient online submission

- thorough peer review by experienced researchers in your field

- rapid publication on acceptance

- support for research data, including large and complex data types

- gold Open Access which fosters wider collaboration and increased citations

- maximum visibility for your research: over $100 \mathrm{M}$ website views per year

At BMC, research is always in progress.

Learn more biomedcentral.com/submissions 